\title{
The basics and issues of Thermochromic Liquid Crystal Calibrations.
}

\begin{abstract}
Thermochromic Liquid Crystals (TLCs) have been widely used by researchers in heat transfer and fluid flow communities as a thermal imaging tool for mapping surface and spatial temperature distributions. In order to utilize TLCs for quantitative temperature measurements, calibration is first necessary to determine the colour-temperature relationship of TLCs. This paper is aimed to provide novice and intermediate users of TLCs with a review on the basics and issues pertaining to calibrations of TLCs, particularly for surface thermography. A general overview of TLCs, the basic elements of a TLC calibration rig, and the common calibration methods of TLCs are described. The crucial issues associated with calibrations of TLCs, namely, imaging, colourimetry, illumination, hysteresis, film thickness and aging, and the methods used to compensate for these effects are discussed. This paper is intended to provide useful information to novice and intermediate users of TLCs, particularly on TLC calibrations.
\end{abstract}

Keyword: Calibration; Colour space; Heat transfer; Liquid Crystal Thermography; Thermochromic Liquid Crystals. 